\title{
Folliculitis decalvans mimicking lupus vulgaris successfully treated with minocycline and clarithromycin
}

\author{
Shivani Ranjan ${ }^{1, *}$, Reeta Gupta Sood ${ }^{2}$ \\ ${ }^{\mathbf{1}}$ Senior Resident, ${ }^{2}$ Professor, Dept. of Dermatology, Veneorology, Leprology, ASCOMS \& Hospital, Sidhra, Jammu, Jammu and \\ Kashmir, India \\ *Corresponding Author: \\ Email: drshivaniranjan@ rocketmail.com
}

\begin{abstract}
Folliculitis decalvans is recurrent purulent folliculitis resulting in cicatrizing alopecia. We report a case of 52 years old male presented with folliculitis decalvans mimicking lupus vulgaris successfully treated with minocycline and clarithromycin.
\end{abstract}

Keywords: Folliculitis decalvans, Cicatricial alopecia, Lupus vulgaris, Minocycline, Clarithromycin.

\section{Introduction}

Folliculitis decalvans (FD) also known as Quinquad's disease was first described by Quinquad in 1888. ${ }^{1}$ Decalvans is a term derived from latin meaning "making bald". It is a neutrophilic primary cicatricial alopecia. Approximately, $11 \%$ of all primary cicatricial alopecias are diagnosed with $\mathrm{FD}^{2,3}$ It predominantly occurs in young and middle aged adults with a slight preference of male gender. It frequently starts at the vertex and occipital areas of the scalp. The aetiology of FD is not fully understood. A bacterial infection involving Staphylococcus aureus, in combination with hypersensitivity reaction to "superantigens" and defect in host cell-mediated immunity have all been suspected as possible pathogenetic factors. ${ }^{2,-6}$

\section{Case Report}

52 years old male presented with recurrent inflammatory lesions over scalp since 6 years. Lesion started as a papulopustule associated with itching and oozing of pus and sometimes blood followed by loss of hair. The lesion gradually increased in size. History of few discrete pustular lesions in the other areas of scalp also present. The patient had received many treatments with disappointing results. On examination, single well defined alopecic plaque measuring about $6 \times 5 \mathrm{~cm}$ in size showing hemorrhagic adherent crusting in the centre, scarring at upper end and progression at the lower end. Clinically it mimicked lupus vulgaris but on close examination, there was presence of tufted follicles over lower end of plaque and active follicular pustules with yellowish crusting and matting of hair in the periphery of lower portion of plaque. Also other areas of scalp showed presence of few discrete follicular papular and pustular lesions. So skin biopsy was done keeping folliculitis decalvans and dissecting folliculitis of scalp as impressions. Skin biopsy confirmed the diagnosis of late stage of folliculitis decalvans showing presence of perifollicular fibrosis and inflammation composed predominantly of lymphocytes and few plasma cells. The patient was put on minocycline 100 $\mathrm{mg}$, clarithromycin $500 \mathrm{mg}$ orally and clindamycin $1 \%$ gel locally. Patient started showing improvement after 1 week of treatment. After 3 months, clarithromycin was stopped and now patient is maintaining on minocyline orally and clindamycin locally for the last 3 months with no evidence of relapse.
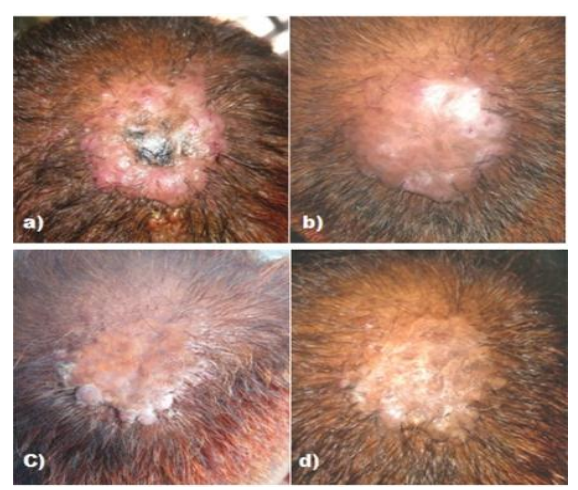

Fig. 1 a: Baseline, note the scarring at upper end and progression at lower end mimicking lupus vulgaris; tufted follicles and active pustules towards the lower portion of plaque. Fig. b,c,d: showing improvement with treatment at 2 weeks, 3 months, 6 months

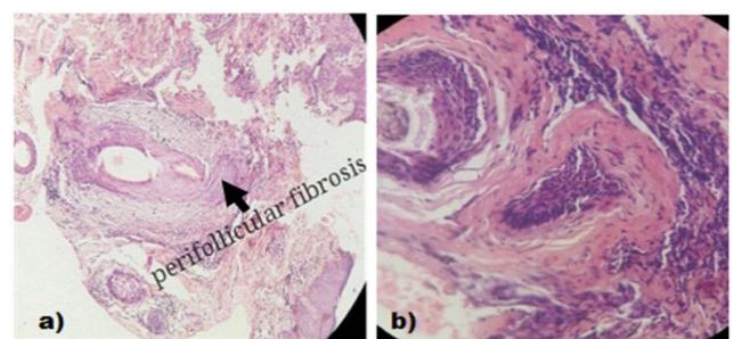

Fig. 2 a: Low power view showing perifollicular fibrosis and inflammatory cell infiltrate Fig. b: High power view showing perifollicular fibrosis and inflammatory cell infiltrate rich in lymphocytes and few plasma cells 


\section{Discussion}

FD presents with follicular papulo-pustules, follicular scarring, perifollicular erythema and scaling, follicular tufting, and sometimes, hemorrhagic crusts and erosions. Usually asymptomatic, patients may complain of pain, itching, burning sensation, spontaneous bleeding and pus discharge. On histopathology, in early lesions there is a perifollicular infiltrate rich in neutrophils. The infiltrate develops into perifollicular abscess leading to destruction of hair and hair follicles. Older lesions show chronic granulation tissue containing numerous plasma cells, lymphocytes and fibroblasts. As healing takes place, fibrosis is observed.

Therapeutic options include oral and topical antimicrobials, oral and topical corticosteroids, oral isotretinoin, dapsone, oral zinc sulfate and oral fusidic acid, laser epilation with Nd: YAG laser, scalp reduction and hair transplantation. ${ }^{6}$ Clindamycin and rifampicin due to their excellent antistaphylococcal activity, have been reported most successful. ${ }^{4,7}$ However, this combination carries a risk of adverse effects. Also, use of rifampicin may provoke resistance. ${ }^{4}$ Sillani et $\mathrm{al}^{8}$ suggested the use of minocycline in mild cases, and combination of Rifampicin or Clarythromycin with Minocycline in moderate or resistant cases.

Minocycline inhibits protein synthesis of Gram negative and Gram positive microorganisms, including $S$. aureus. Clarythromycin, a macrolide antimicrobial, binds to the $50 \mathrm{~S}$ ribosomal subunit of susceptible microorganisms and inhibits protein synthesis. We have used clarithromycin and minocyline combination and found excellent results with rapid remission. Also, our patient didn't not notice any side effects with the treatment.

\section{Conclusion}

FD is a very distressing disease both for the patient as well as the treating physician because of the long lasting waxing and waning course, the resultant cicatricial alopecia and long term treatment. Despite a plethora of treatments claimed to be effective, we usually observe a lack of response because of the unclear aetiology and pathogenesis. In our opinion, the combination of minocycline with clarithromycin is a good candidate for substituting rifampicin and clindamycin combination with comparable treatment outcome and lesser side effects.

\section{References}

1. Quinquad E. Folliculite destructive des regions values. Bull. Et mem de la soc. Hop de paris. 1888;5:395-398

2. Whiting DA. Cicatricial alopecia: clinico-pathological findings and treatment. Clin Dermatol 2001:19:211215.

3. Tan E, Martinka M, Ball N, Shapiro J. Primary cicatricial alopecias: clinicopathology of 112 cases. J Am Acad Dermatol 2004:50:25-32.
4. Powell JJ, Dawber RPR, Gatter K. Folliculitis decalvans including tufted folliculitis: clinical, histological and therapeutic findings. Br J Dermatol 1999; 140:328-33.

5. Powell J DR: Successful treatment regime for folliculitis decalvans despite uncertainty of all aetiological factors. Br J Dermatol 2001;144:428-429,

6. Otberg N, Kang H, Abdullateef A. Alzolobani \& Jerry S. Folliculitis decalvans. Dermatologic Therapy.2008; 21:238-244

7. Pratenda G, Grimaldi M, Palese D, Di Napoli A, Bottoni U. Tufted hair folliculitis: complete enduring response after treatment with rifampicin. J Dermatolog Treat 2004; 15:396-8.

8. Sillani C, Bin Z, Ying Z, et al. Effective treatment of Folliculitis Decalvans using selected antimicrobial agents. Int J Trichology. 2010 Jan-Jun; 2(1): 20-23.4. 